\title{
Phototropism of Conidial Germ Tubes of Botrytis cinerea and Its Implication in Plant Infection Processes
}

S. Z. Islam, Graduate Student, and Y. Honda, Professor, Faculty of Life and Environmental Science; and M. Sonhaji, Graduate Student, Interdisciplinary Faculty of Science and Engineering, Shimane University, Matsue, Shimane 690-8504, Japan

\begin{abstract}
Islam, S. Z., Honda, Y., and Sonhaji, M. 1998. Phototropism of conidial germ tubes of Botrytis cinerea and its implication in plant infection processes. Plant Dis. 82:850-856.

The germ tubes of Botrytis cinerea showed negative phototropism to near ultraviolet (NUV) and blue (300 to $520 \mathrm{~nm}$ ) light followed by far-red (700 to $810 \mathrm{~nm}$ ), whereas red light (600 to 700 $\mathrm{nm}$ ) induced positive phototropism significantly. Minimum germ tube growth occurred during exposure to negative phototropism-inducing wavelengths, whereas it was maximum under positive phototropism-inducing wavelengths. NUV radiation and blue light that induced negative phototropism of $B$. cinerea promoted infection-hypha formation on both onion scale and broad bean (Vicia faba) leaf epidermal strips, whereas positive phototropism-inducing red light suppressed it, resulting in a high proportion of germ tubes without infection hyphae. In broad bean leaf infection, the number of infection points and area of necrosis per drop of conidial suspension were higher under NUV radiation and blue light than that of a dark control or leaflets pretreated with NUV radiation and blue light. In contrast, lower numbers of infection points and very small necrotic lesions developed under red light. In the case of red-light-pretreated leaflets, the number of infection points developed were higher, but areas of necrosis did not increase significantly compared with leaflets kept under red light without pretreatment. These results show the importance of phototropism of conidium germ tubes in plant infection.
\end{abstract}

The lives of fungi are greatly influenced by light, as seen in the formation of reproductive structures, pigment biosynthesis, phototaxis, and phototropism $(12,18)$. Phototropism of conidium germ tubes of plant pathogenic fungi is important in the plant infection process $(5,8,10,21)$. Close contact or adhesion of fungal-conidium germ tubes to a host surface is essential for infection structure initiation (3,14-17). Negative phototropism facilitates the contact of the tips of conidium germ tubes with the host surface that subsequently promotes infection structure formation and favors the infection process $(4,8,10,21)$. Koch and Hoppe (10) studied the doseresponse curves for negative phototropism of uredospore germ tubes of Puccinia pachyrhizi at several wavelengths ranging from 372 to $600 \mathrm{~nm}$ and concluded that negative phototropism of germ tubes was a blue-light effect, and that wavelengths longer than $600 \mathrm{~nm}$ (red light) were ineffective in phototropism. Honda and Miya-

Corresponding author: Yuichi Honda

E-mail: honda@life.shimane-u.ac.jp

Accepted for publication 30 April 1998.

Publication no. D-1998-0608-02R

(C) 1998 The American Phytopathological Society waki (6) showed that, in Septoria obesa, negative phototropism of germ tubes was induced by radiation between 300 and 520 $\mathrm{nm}$, with maxima at $380 \mathrm{~nm}$ in the near ultraviolet (NUV) region and $460 \mathrm{~nm}$ in the blue region, and that radiation of 580 to $700 \mathrm{~nm}$ induced positive phototropism. Recently, Islam and Honda (8) showed that, in Colletotrichum lagenarium, negative phototropism of germ tubes was induced by UV to blue ( 300 to $520 \mathrm{~nm}$ ) and far-red $(>700 \mathrm{~nm})$ light with a maximum in the NUV region, whereas wavelengths of 575 to $700 \mathrm{~nm}$ (yellow-red region) induced positive phototropism with a maximum in the red region.

Honda et al. (5) showed that elimination of negative phototropism-inducing wavelengths shorter than $390 \mathrm{~nm}$ by an ultraviolet-absorbing (UVA) vinyl film reduced the invasion of conidium germ tubes of $S$. obesa through the stomata. They also showed that, in greenhouse experiments, the number of brown spot lesions on chrysanthemum leaves in the greenhouse under UVA vinyl film was lower than the number of leaf spots on plants under common agricultural (CA) vinyl film. In our previous report (8), we showed that appressorium formation of $C$. lagenarium was promoted significantly by negative phototropisminducing NUV radiation and it was inhibited significantly by positive phototropism- inducing red light. On the other hand, conidial germ tubes of Bipolaris oryzae did not show a significant phototropic response to light of different wavelengths, and light did not affect the formation of infection structures. The purpose of this study was to examine the phototropic response of conidium germ tubes of Botrytis cinerea Pers.:Fr. to different wavelengths of light, and its implication in plant infection.

\section{MATERIALS AND METHODS}

Culture of plants. Broad bean (Vicia faba L.) plants were grown in a glasshouse throughout the experiment. Seeds were sown in $16-\mathrm{cm}$-diameter pots containing 3 $\mathrm{kg}$ of commercial garden soil (Kureha Chemicals, Osaka, Japan), 1 seed/pot.

Pathogen. Isolates of $B$. cinerea, two from strawberries $(\mathrm{BC} 1$ and $\mathrm{BC} 2)$ and two from green peppers (BC3 and $\mathrm{BC} 4)$, were cultured on potato sucrose agar (PSA) medium at $22.5^{\circ} \mathrm{C}$ (7) under continuous NUV irradiation provided by fluorescent lamp $\left(360 \mu \mathrm{W} / \mathrm{cm}^{2}\right.$; FL10BLB, Toshiba, Tokyo, Japan). A lawn of uniform sporula-

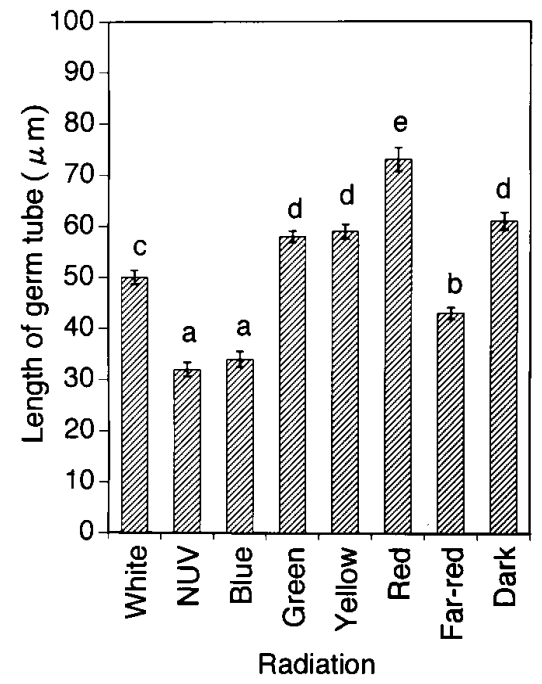

Fig. 1. Effect of light on conidium germ tube growth of Botrytis cinerea; isolate BC1. Germ tubes were measured after $12 \mathrm{~h}$ of irradiation. Bars in the columns show the standard errors. Means followed by the same letter are not significantly different at $P<0.05$ according to Duncan's multiple range test. 
tion was formed within 5 to 6 days. A conidial suspension was prepared from conidia dislodged with a glass rod in plates of 7-day-old cultures flooded with sterilized, distilled water. Conidial densities were estimated with a hemacytometer and adjusted to 1 to $2 \times 10^{5}$ conidia $/ \mathrm{ml}$. Isolate $\mathrm{BC} 1$ of $B$. cinerea, isolated from strawberry, was used throughout the study unless otherwise noted.

Phototropic response of conidium germ tubes. Conidial suspensions were introduced as $0.2-\mathrm{ml}$ aliquots onto wateragar plates (agar $16 \mathrm{~g}$, water $1,000 \mathrm{ml} ; 10$ $\mathrm{ml} / 6-\mathrm{cm}$ diameter Pyrex petri dish). The plates were shaken gently to disperse the conidia over the whole surface, and the free-surface-water of the plates was dried up by exposure for 10 to $15 \mathrm{~min}$ to air flow on a clean bench. Inoculated plates were incubated at $24 \pm 1^{\circ} \mathrm{C}$ under different wavelengths of light from fluorescent lamps suspended $20 \mathrm{~cm}$ above the plates. Fluorescent lamps provided light in the following ranges: 380 to $740 \mathrm{~nm}$, white light, $473 \mu \mathrm{W} / \mathrm{cm}^{2}$ (FL20SS·D/18, Mitsu- bishi, Tokyo, Japan); 300 to $410 \mathrm{~nm}$, maximum $360 \mathrm{~nm}$, NUV radiation, 374 $\mu \mathrm{W} / \mathrm{cm}^{2}$ (FL20S.BLB, Toshiba); 400 to $520 \mathrm{~nm}$, maximum $450 \mathrm{~nm}$, blue light, 231 $\mu \mathrm{W} / \mathrm{cm}^{2}$ (FL20S·B-F, National, Osaka, Japan); 500 to $575 \mathrm{~nm}$, maximum $540 \mathrm{~nm}$, green light, $184 \mu \mathrm{W} / \mathrm{cm}^{2}$ (FL20S.G-F, National); 540 to $640 \mathrm{~nm}$, maximum 590 $\mathrm{nm}$, yellow light, $320 \mu \mathrm{W} / \mathrm{cm}^{2}$ (FL20S.Y-F, National); 600 to $700 \mathrm{~nm}$, maximum 650 $\mathrm{nm}$, red light, $258 \mu \mathrm{W} / \mathrm{cm}^{2}$ (FL20S.R-F, National); and 700 to $810 \mathrm{~nm}$, maximum $740 \mathrm{~nm}$, far-red light, $288 \mu \mathrm{W} / \mathrm{cm}^{2}$ (FL20S·FR-74, Toshiba). All light intensities were measured through the petri dish. Control plates in light-tight boxes were kept near the test plates at the same conditions. After $10 \mathrm{~h}$ of incubation, inoculated plates were observed under a light microscope to determine the phototropic response of germ tubes. Germ tubes with tips growing away from the light source into the agar surface were considered to be negatively phototropic, and those growing upward from the agar surface toward the light source were positively phototropic.

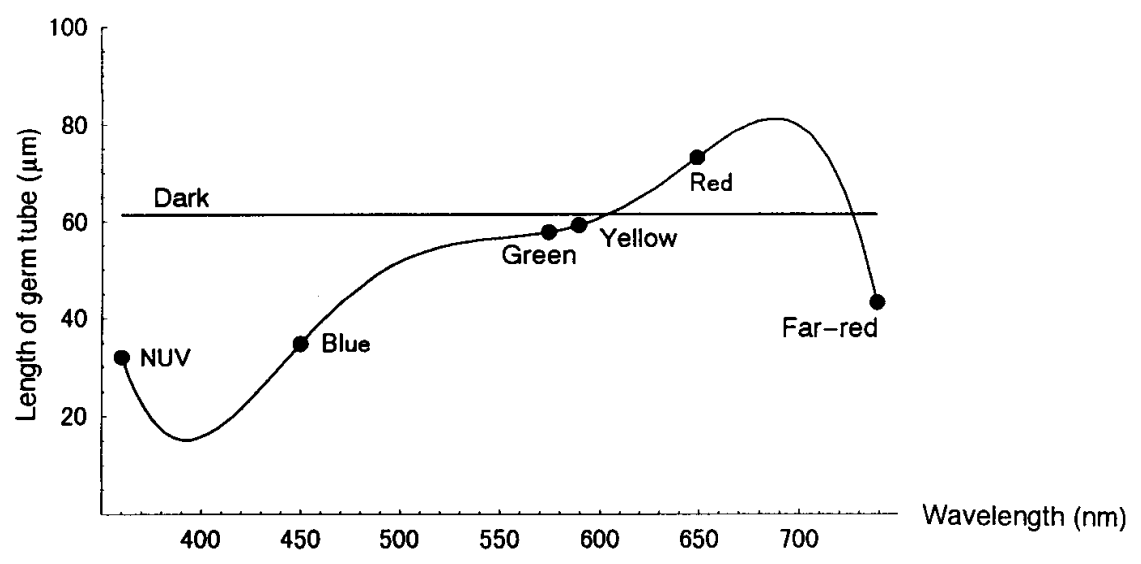

Fig. 2. Interpolating polynomial function analysis of germ tube growth of Botrytis cinerea.

The germ tubes showing no phototropic response were considered to be neutral. Phototropic responses of germ tubes were determined for 200 germ tubes in three to four arbitrarily selected areas of a plate per replication for each isolate, and four plates were examined per treatment. For germ tube growth, 60 germ tubes were measured per replication. Each experiment was repeated twice with four replications. For germ tube growth, analysis of variance (ANOVA) was done to determine the significance of differences between the treatments. To show a relationship between

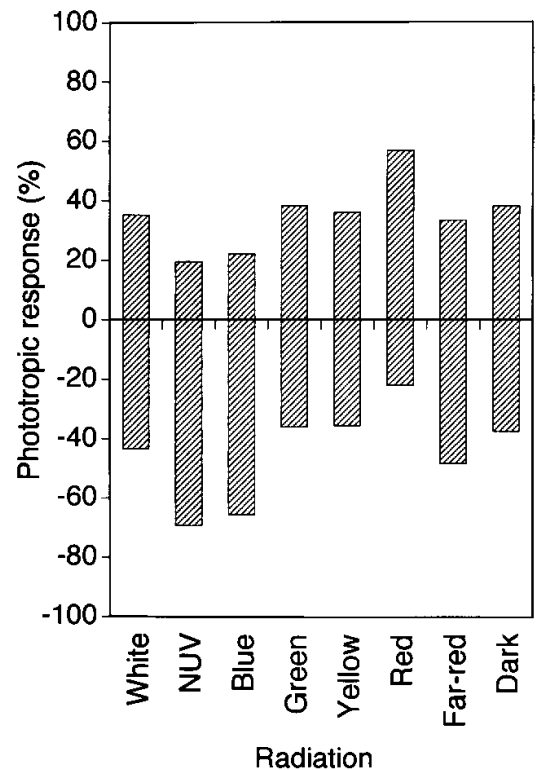

Fig. 3. Phototropic response of conidium germ tubes of Botrytis cinerea. Germ tubes were evaluated after $10 \mathrm{~h}$ of irradiation. Vertical axis shows the percentage of germ tubes that show positive phototropic response $(+)$ and negative phototropic response $(-)$. Bars are the means of four isolates.

Table 1. Phototropic response of conidium germ tubes of Botrytis cinerea in the light of different wavelengths ${ }^{\mathrm{a}}$

\begin{tabular}{|c|c|c|c|c|c|c|c|c|c|c|c|c|c|c|c|c|}
\hline \multirow[b]{2}{*}{ Isolates $^{\mathbf{b}}$} & \multicolumn{2}{|c|}{ White light } & \multicolumn{2}{|c|}{ NUV } & \multicolumn{2}{|c|}{ Blue light } & \multicolumn{2}{|c|}{ Green light } & \multicolumn{2}{|c|}{ Yellow light } & \multicolumn{2}{|c|}{ Red light } & \multicolumn{2}{|c|}{ Far-red } & \multicolumn{2}{|c|}{ Dark } \\
\hline & $(+)$ & $(-)$ & $(+)$ & $(-)$ & $(+)$ & $(-)$ & $(+)$ & $(-)$ & $(+)$ & $(-)$ & $(+)$ & $(-)$ & $(+)$ & $(-)$ & $(+)$ & $(-)$ \\
\hline \multirow[t]{3}{*}{$\mathrm{BC} 1$} & 38 & 46 & 26 & 67 & 28 & 68 & 39 & 36 & 43 & 39 & 58 & 24 & 37 & 52 & 46 & 36 \\
\hline & 42 & 47 & 22 & 70 & 24 & 64 & 42 & 38 & 46 & 37 & 59 & 22 & 35 & 52 & 43 & 39 \\
\hline & 35 & 47 & 25 & 68 & 32 & 62 & 38 & 37 & 40 & 42 & 65 & 19 & 39 & 48 & 44 & 39 \\
\hline Mean & 38.3 & 46.6 & 24.3 & 68.3 & 28.0 & 64.6 & 39.6 & 37.0 & 43.0 & 39.3 & 60.6 & 21.6 & 37.0 & 50.6 & 44.3 & 38.2 \\
\hline \multirow[t]{3}{*}{$\mathrm{BC} 2$} & 31 & 39 & 15 & 55 & 24 & 62 & 36 & 32 & 31 & 32 & 51 & 24 & 28 & 49 & 38 & 36 \\
\hline & 29 & 35 & 19 & 57 & 17 & 72 & 34 & 35 & 36 & 38 & 49 & 14 & 29 & 46 & 35 & 29 \\
\hline & 34 & 37 & 12 & 62 & 22 & 63 & 37 & 29 & 29 & 34 & 52 & 20 & 35 & 39 & 31 & 34 \\
\hline Mean & 31.3 & 37.1 & 15.3 & 58.1 & 21.0 & 65.6 & 35.6 & 32.2 & 32.3 & 34.6 & 50.7 & 19.3 & 30.6 & 44.6 & 34.6 & 33.1 \\
\hline \multirow[t]{3}{*}{$\mathrm{BC} 3$} & 37 & 49 & 17 & 67 & 21 & 73 & 45 & 39 & 38 & 36 & 66 & 27 & 32 & 49 & 35 & 36 \\
\hline & 33 & 43 & 25 & 79 & 23 & 61 & 39 & 42 & 41 & 35 & 62 & 24 & 29 & 56 & 39 & 42 \\
\hline & 36 & 47 & 22 & 73 & 16 & 65 & 41 & 35 & 34 & 32 & 52 & 21 & 34 & 46 & 37 & 43 \\
\hline Mean & 35.3 & 46.3 & 21.3 & 73.1 & 20.0 & 66.3 & 41.6 & 38.6 & 37.6 & 34.3 & 60.2 & 24.1 & 31.6 & 50.3 & 37.1 & 40.3 \\
\hline \multirow[t]{3}{*}{$\mathrm{BC} 4$} & 35 & 41 & 20 & 77 & 25 & 60 & 39 & 36 & 35 & 38 & 54 & 23 & 34 & 48 & 41 & 45 \\
\hline & 39 & 44 & 15 & 76 & 15 & 70 & 32 & 37 & 31 & 29 & 52 & 20 & 32 & 52 & 34 & 37 \\
\hline & 34 & 47 & 16 & 81 & 18 & 65 & 37 & 35 & 29 & 33 & 60 & 25 & 37 & 43 & 35 & 33 \\
\hline Mean & 36.1 & 44.2 & 17.1 & 78.0 & 19.4 & 65.0 & 36.0 & 36.0 & 31.6 & 33.3 & 55.3 & 22.6 & 34.3 & 47.6 & 36.6 & 38.3 \\
\hline Gross mean ${ }^{\mathrm{c}}$ & 35.3 & 43.5 & 19.5 & 69.3 & 22.2 & 65.5 & 38.3 & 35.9 & 36.1 & 35.4 & 57.0 & 22.0 & 33.4 & 48.3 & 38.2 & 37.4 \\
\hline
\end{tabular}

${ }^{a}(+)$ denotes positive phototropic response (\%); (-) denotes negative phototropic response (\%).

${ }^{b}$ The rest of percentage of germ tubes of each isolate for each treatment are neutral.

${ }^{c}$ Average of means of four isolates. 
wavelengths and germ tube growth, polynomial analysis was also done (2). For phototropic responses of germ tubes, we analyzed negative phototropic responses of germ tubes by polynomial analysis to establish a relationship between phototropic responses of germ tubes and wavelengths.

Formation of infection hypha under negative phototropism-inducing NUV radiation and blue light and positive phototropism-inducing red light. Both onion scale and broad bean leaf epidermal strips were used in this experiment. Epidermal strips were used to examine the effect of phototropic responses of germ tubes on infection hypha formation without any intervention of host resistance. The method used to observe infection hyphae on onion epidermis was described previously (8). Small strips $(1.5$ by $1.5 \mathrm{~cm})$ of leaf epidermis were peeled from the upper surfaces of broad bean leaflets. The strips were placed cuticle side up on sterilized glass slides in petri dishes lined with sterilized moist filter paper. A conidial suspension $\left(50 \mathrm{ml}, 2 \times 10^{5}\right.$ conidia $\left./ \mathrm{ml}\right)$ was dispensed to the center of each epidermal strip and incubated under light of different wavelengths at $24 \pm 1^{\circ} \mathrm{C}$. Epidermal strips in petri dishes kept in light-tight boxes were incubated as a control near the test petri dishes. Infection hyphae were observed after $12 \mathrm{~h}$ of incubation. Germ tubes with and without infection hyphae were counted as infection hyphae and germ tubes, respectively. An infection hypha was defined as a hypha which was formed inside the host cell or tissue from the appressorium. To facilitate observation, fungal structures were stained with a solution consisting of $0.2 \%$ trypan blue, $20 \%$ melted phenol (vol/vol), 20\% lactic acid (vol/vol), $40 \%$ glycerol (vol/vol), and $20 \%$ water (20). Two epidermal strips were used per replication. For each replication, 180 to 200 germ tubes were evaluated, and each experiment was repeated three times with three replications. Data were subjected to ANOVA followed by Dunnett's test for means comparison.
Infection of leaflets exposed to NUV radiation, blue light, and red light. The third fully expanded bifoliate leaves from the tops of the broad bean plants were used in these experiments. One leaflet of a bifoliate leaf was used for control and the other was used for treatment. Detached leaflets were washed in a stream of distilled water and allowed to dry before inoculation. Leaflets were placed on a frame of glass rods in petri dishes lined with sterilized moist filter paper. Drops $(10 \mu \mathrm{l})$ of conidial suspension $\left(1 \times 10^{5}\right.$ conidia $\left./ \mathrm{ml}\right)$ taken from a beaker kept on an electric stirrer were put on the upper surfaces of the leaflets (6 to 8 drops per leaflet) by a micropipette and incubated under the light of different wavelengths at $24 \pm 1{ }^{\circ} \mathrm{C}$. One leaflet or half-leaflet was considered as one replication. The number of infection points were counted after $24 \mathrm{~h}$ of incubation, and the area of necrosis was measured after $48 \mathrm{~h}$ of incubation. We diagnosed the infection points by examining the browning of epidermal cells around the appressoria under a compound microscope. Necrotic lesions in an enlarged photograph of infection points were traced on tracing-paper, and the areas of necrosis were computed with Adobe Photoshop 2.5 J and NIH image software. At first, we counted the number of infection points after 24 and $48 \mathrm{~h}$, but as there were rarely any significant differences between the two counts, the second count was omitted. Each experiment was repeated three times with four replications. The data for number of infection points and area of necrosis were analyzed separately by ANOVA followed by Duncan's multiple range test for means comparison. Analysis of data was also done by polynomial analysis to show a relationship between leaf infection and wavelengths.

Pretreatment of leaflets with light. We carried out pretreatment of leaves to distinguish the effect of light on the host leaf from the effect of phototropism of germ tubes in infection process. Detached broad bean leaflets, placed on glass rod frames in

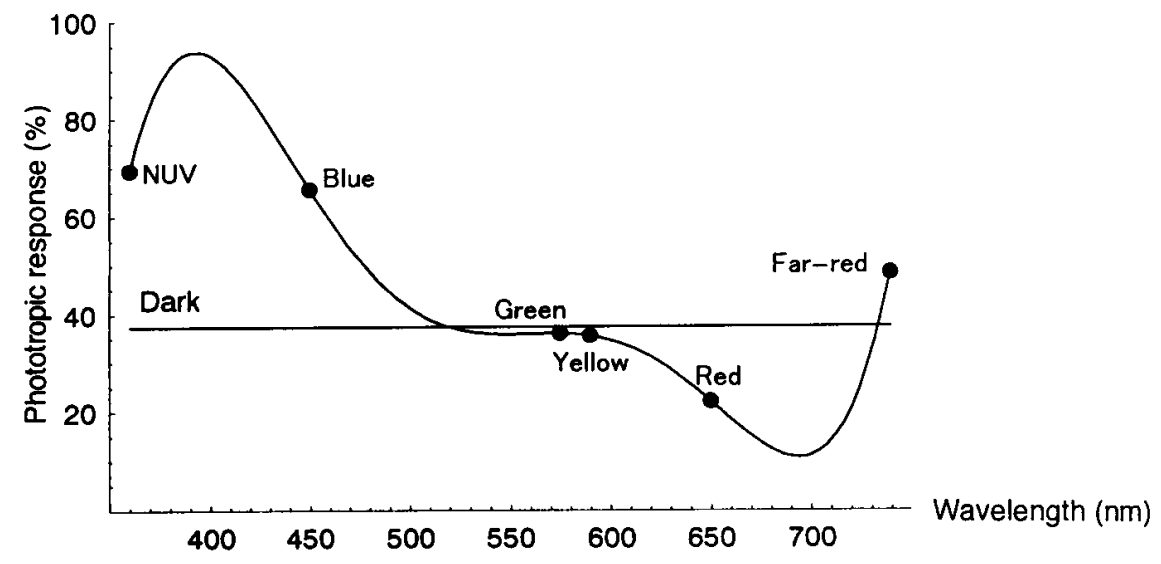

Fig. 4. Interpolating polynomial function analysis of negative phototropic response of germ tubes of Botrytis cinerea. petri dishes lined with sterilized moist filter paper, were pretreated for $24 \mathrm{~h}$ with light of different wavelengths. After that, the leaflets were inoculated with $10-\mu \mathrm{l}$ drops of a $10^{5}$ conidia/ml suspension and kept in dark boxes at $24 \pm 1^{\circ} \mathrm{C}$. At the same time, the control leaflets were pretreated by incubation in darkness for $24 \mathrm{~h}$ and then inoculated and kept in dark boxes near the test leaflets. One leaflet was considered as one replication and each experiment was repeated three times with four replications. The infection points were counted after $24 \mathrm{~h}$ of incubation, and the area of necrosis was measured after $48 \mathrm{~h}$ of incubation. ANOVA were performed as mentioned above for post-treatment.

\section{RESULTS}

Effect of light on conidium germ tube growth. Minimum germ tube growth occurred in NUV radiation and blue light followed by far-red and white light. On the other hand, maximum germ tube growth occurred in red light. Yellow and green light did not affect germ tube growth significantly compared with the dark control (Fig. 1). By interpolating polynomial analysis, we got the equation $Y=25329.9$ $-2244.621 x+0.931104 x^{2}-0.00174664 x^{3}$ $+1.61731 \times 10^{-6} x^{4}-5.91866 \times 10^{-10} x^{5}$, where $Y$ is germ tube growth and $x$ is wavelength. This equation shows a relationship between germ tube growth and wavelengths (Fig. 2). Minimum germ tube growth was observed under wavelengths of 300 to $520 \mathrm{~nm}$ (NUV to blue region) that induced negative phototropism, whereas maximum germ tube growth was under positive phototropism-

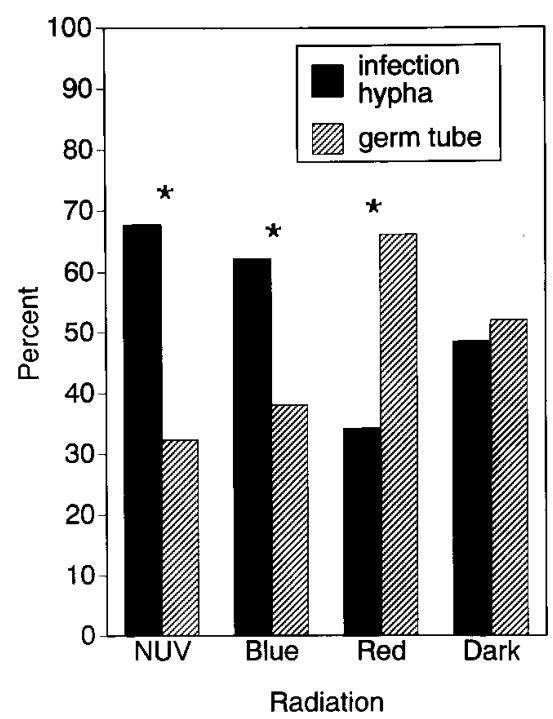

Fig. 5. Effect of light on infection hypha formation of Botrytis cinerea on broad bean (Vicia faba L.) leaf epidermis after $12 \mathrm{~h}$ of irradiation. Differences between infection hypha and germ tube formation were compared between light treatments and a dark control according to Dunnett's test. Asterisks denote significant differences at $P<0.05$. 
inducing wavelengths of 600 to $700 \mathrm{~nm}$ (red region).

Phototropic response of conidium germ tubes. All the isolates of $B$. cinerea showed a strong negative phototropic response in NUV radiation and blue light and a strong positive phototropic response in red light (Table 1, Fig. 3). Under NUV radiation and blue light, 65.5 to $69.3 \%$ of germ tubes were negatively phototropic, 19.5 to $22.2 \%$ were positively phototropic, and 11.2 to $12.3 \%$ were neutral. In contrast, under red light, $57 \%$ of germ tubes were positively phototropic, $22 \%$ were negatively phototropic, and $21 \%$ were neutral. In far-red light, $48.3 \%$ of germ tubes were negatively phototropic, $33.4 \%$ were positively phototropic, and $18.3 \%$ were neutral. White light induced relatively weak negative phototropic response, under which $43.5,35.3$, and $21.2 \%$ of germ tubes showed negative, positive, and neutral responses, respectively.

Analysis of negative phototropic responses of germ tubes by polynomial analysis resulted in the equation $\mathrm{Y}=$ $-34714.9+334.377 \mathrm{x}-1.26406 \mathrm{x}^{2}+$ $0.00235281 x^{3}-2.15989 \times 10^{-6} x^{4}+$ $7.83162 \times 10^{-10} X^{5}$, where $Y$ is phototropic response and $X$ is wavelength (Fig. 4). It shows that NUV to blue light is most effective for negative phototropic response of germ tubes of $B$. cinerea.

Infection hypha formation on onion and leaf epidermal strips. NUV radiation and blue light significantly promoted the formation of infection hyphae. In contrast, red light inhibited infection hypha formation significantly and increased the percentage of germ tubes without infection hyphae over that in the dark control (Fig. 5).

Infection of leaflets under the light of different wavelengths. Under NUV radiation and blue light, which induced a strong negative phototropic response of germ tubes of $B$. cinerea, the number of infection points developed per drop of conidial suspension was significantly $(P=$ $0.05)$ higher than red light treatment and dark control. In contrast, under red light, which induced a strong positive phototropic response, the number of infection points developed was significantly lower than that of NUV radiation, blue light, and dark control (Figs. 6, 7A to D). Polynomial analysis of the number of infection points resulted in the equation $Y=10.239+$ $1.25103 x-0.00186685 x^{2}$, where $Y$ is number of infection points and $X$ is wavelength, which shows a relationship between number of infection points and wavelength. Larger necrotic areas developed per drop of conidial suspension under both NUV radiation and blue light compared with red light or dark control. In the case of red light, very small necrotic lesions developed (Figs. 8, 9A to D). Polynomial analysis of necrosis developed resulted in the equation $Y=-26.2954+$
$0.254288 x-0.000320383 x^{2}$, where $Y$ is area of necrosis and $X$ is wavelength.

Pretreatment of leaflets with light. In the case of pretreatment of leaflets with both NUV radiation and blue light, the number of infection points developed per drop of conidial suspension was significantly $(P=0.05)$ lower than that for leaf-

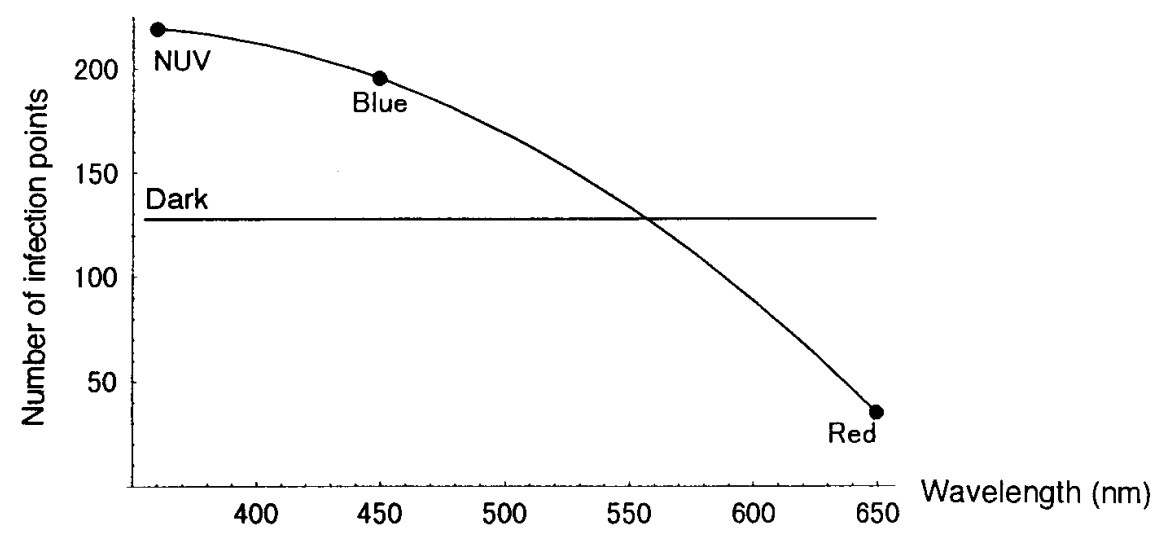

Fig. 6. Interpolating polynomial function analysis of infection points developed per drop of inoculated conidial suspension after $24 \mathrm{~h}$ of inoculation in broad bean leaflets by Botrytis cinerea under light (post-treatment) of different wavelengths.
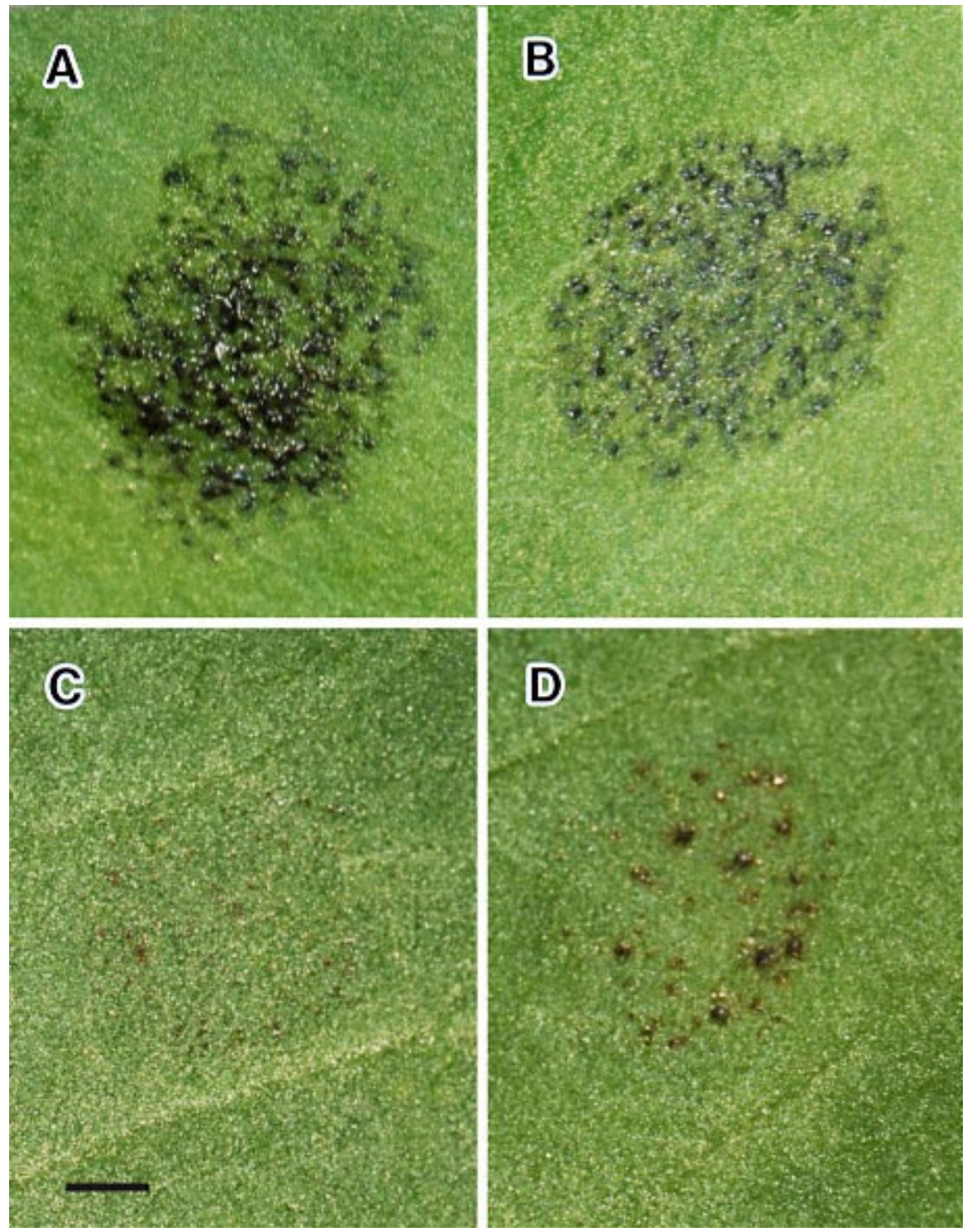

Fig. 7. Infection points of Botrytis cinerea developed per drop of inoculated conidial suspension on broad bean leaflet after $24 \mathrm{~h}$ of inoculation (A) under NUV radiation, (B) under blue light, (C) under red light, and (D) under dark. Scale bar $=1 \mathrm{~mm}$. 
lets kept under NUV radiation or blue light without pretreatment (Fig. 10). In redlight-pretreated leaflets, the number of infection points per drop of conidial suspension was significantly higher than that for leaflets kept under red light without pretreatment. Polynomial analysis of the number of infection points resulted in the equation $Y=160.585+0.0192958 x$ - $0.000243301 X^{2}$, where $Y$ is number of infection points and $X$ is wavelength. The necrotic area developed per drop of conidial suspension was significantly smaller in both NUV radiation and blue-light-pretreated leaflets than in dark-pretreated leaves or leaflets without pretreatment with NUV radiation and blue light, respectively. In red-light-pretreated leaflets, the area of necrosis was not significantly larger than in the leaflets kept under red light without pretreatment (Figs. 11, 12A to D). We got the equation $Y=-21.2216+0.157404 x$ - $0.000180792 x^{2}$ by polynomial analysis of area of necrosis, where $Y$ is area of necrosis and $X$ is wavelength.

Germ tube phototropism of $B$. cinerea in leaf infection. The number of infection points and area of necrosis developed in the light of different wavelengths (Figs. 6 and 8 ) is the combined effect of both germ tube phototropism and light effect on host. Therefore, we carried out pretreatment of leaves to separate the effect of germ tube phototropism in the infection process.

We calculated the number of infection points and area of necrosis by deduction of data of pretreatment from post-treatment of leaves. This resultant number of infection points and area of necrosis were assumed to be the actual effects of germ tube phototropism in leaf infection. Polynomial analysis of infection points resulted in the equation $Y=-150.35+1.23175 \mathrm{X}-$ $0.00162356 X^{2}$, where $Y$ is number of infection points and $X$ is wavelength (Fig. 13). Under negative phototropism-inducing NUV to blue light, the number of infection points and area of necrosis were significantly $(P=0.05)$ higher than those under red light, which caused a strong positive phototropic response of germ tubes. For area of necrosis, we got the equation $Y=-$ $5.07384+0.0968839 x-0.000139591 x^{2}$, where $Y$ is area of necrosis and $X$ is wavelength (Fig. 14).

\section{DISCUSSION}

All isolates showed a strong negative phototropic response to NUV to blue light followed by far-red light, and a strong positive phototropic response to red light. This result showed that wavelengths of 300 to $520 \mathrm{~nm}$ (UV to blue regions) and of 700 to $810 \mathrm{~nm}$ (far-red region) were effective for negative phototropism of germ tubes of B. cinerea, and wavelengths of 600 to 700 $\mathrm{nm}$ (red region) were effective for positive phototropism. These findings are in general agreement with Honda and Miyawaki (6) and Islam and Honda (8). Jaffe and Etzold (9) also reported that the negative phototropism of conidium germ tubes of $B$. cinerea is due to the effect of blue light, but they did not report in detail about phototropism under different wavelengths. The negative phototropism of germ tubes under white light may be due to the greater amount of negative phototropism-inducing components. The tendency of phototropic responses in all isolates are the same, although some differences exist among isolates.

Blaauw (1) coined the term "light growth reaction" to describe the response of growth acceleration of sporangiophores in Phycomyces spp. induced by irradiation. $\mathrm{He}$ postulated that the "cylindrical lens action" of a sporangiophore is responsible for the influence rate being higher on the distal than proximal side of the sporangiophore. In this mechanism, the growing zone acts as a convergence lens, and thus the distal side of the sporangiophore receives a more intense light stimulus than the proximal side, resulting in greater growth on the distal than proximal side and positive phototropism of the sporangiophore. This hypothesis was supported by the finding that when a sporangiophore was immersed in a medium of higher re-

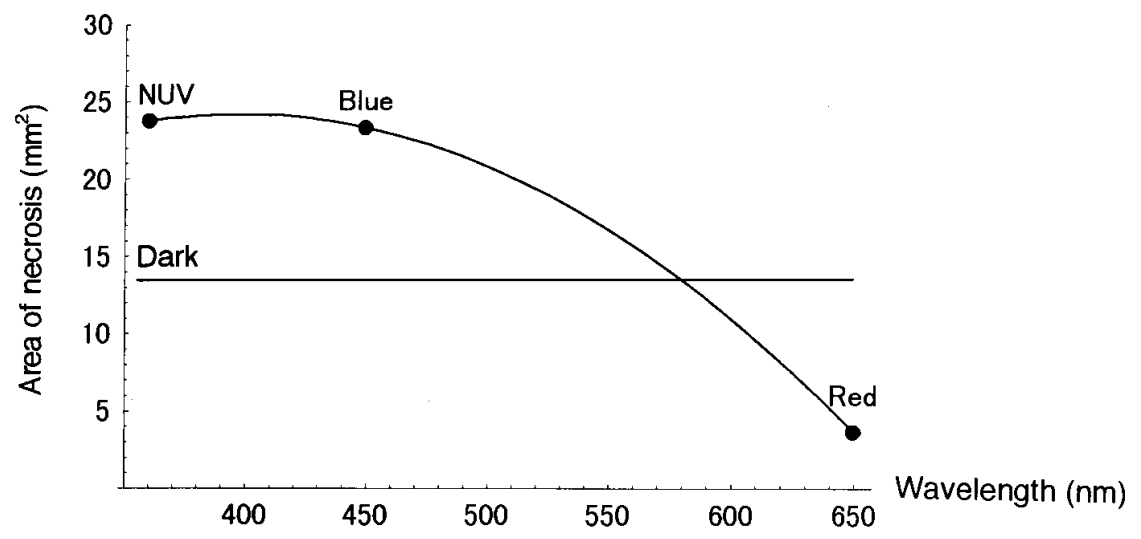

Fig. 8. Interpolating polynomial function analysis of necrosis development of Botrytis cinerea per drop of inoculated conidial suspension in broad bean leaflets after $48 \mathrm{~h}$ of inoculation under light (post-treatment) of different wavelengths.

fractive index than air (e.g., liquid paraffin), the cell became a diverging lens and phototropic bending was reversed.

Maximum germ tube growth of $B$. cinerea occurred in positive phototropism-inducing wavelengths of 600 to $700 \mathrm{~nm}$ (red region). In contrast, minimum germ tube growth occurred in negative phototropisminducing wavelengths of 300 to $520 \mathrm{~nm}$ (NUV to blue regions) followed by 700 to $810 \mathrm{~nm}$ (far-red region). These results are consistent with the previous findings of Honda and Miyawaki (6), Islam and Honda (8), and Koga et al. (11). Germ tubes of Bipolaris oryzae did not show any phototropic response and light did not affect germ tube growth (8). These results suggest the possibility that the lens effect in combination with the light growth reaction (1) and light growth suppression is the mechanism of the phototropism of germ tubes in $B$. cinerea.

On both onion scale and leaf epidermal strips, negative phototropism-inducing NUV radiation and blue light promoted infection hypha formation. On the other hand, red light that induced positive phototropism reduced it significantly. This result is in accordance with the findings of Islam and Honda (8), in which negative phototropism-inducing NUV radiation promoted the infection structure formation of $C$. lagenarium and positive phototropism-inducing red light reduced it significantly.

We used bifoliate leaves of broad bean in these experiments. We also used halfleaflets by dividing one leaflet along its midrib. We did not see any significant difference between leaflets of a bifoliate leaf and between half-leaflets of a leaflet with regard to infection by $B$. cinerea in accordance with the reports of Last and Hamley (13) and Wilson (19). Under the negative phototropism-inducing radiation (NUV and blue), both the number of infection points and area of necrosis developed per drop of inoculated conidial suspension were significantly higher compared with those under positive phototropism-inducing red light. Negative phototropism-inducing NUV radiation and blue light might improve the contact of larger number of tips of the germ tubes with the leaf surfaces. This theory is supported by the fact that, due to the contact stimuli of larger number of tips of the germ tubes with the leaf surfaces, more infection structures were formed, which resulted in an increased number of infection points on broad bean leaflets under NUV radiation and blue light. The opposite occurs under positive phototropisminducing red light.

The higher number of infection points and larger necrotic area have been observed in dark-pretreated leaflets, which may be ascribed to deterioration of host resistance due to longer periods of darkness. In red-light-pretreated leaflets, the 

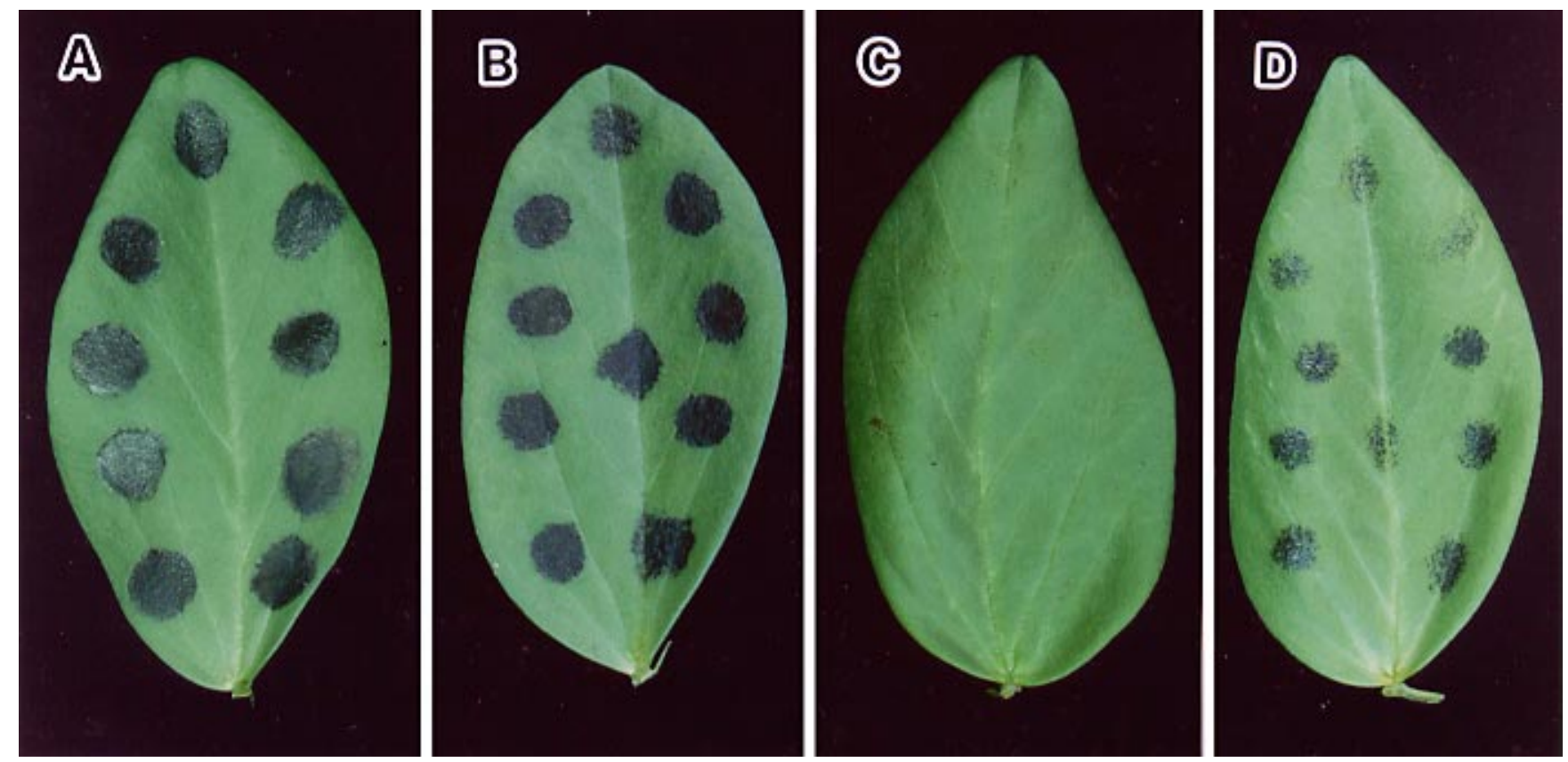

Fig. 9. Necrosis development of Botrytis cinerea in broad bean leaflets after $48 \mathrm{~h}$ of inoculation (A) under NUV radiation, (B) under blue light, (C) under red light, and (D) under dark.

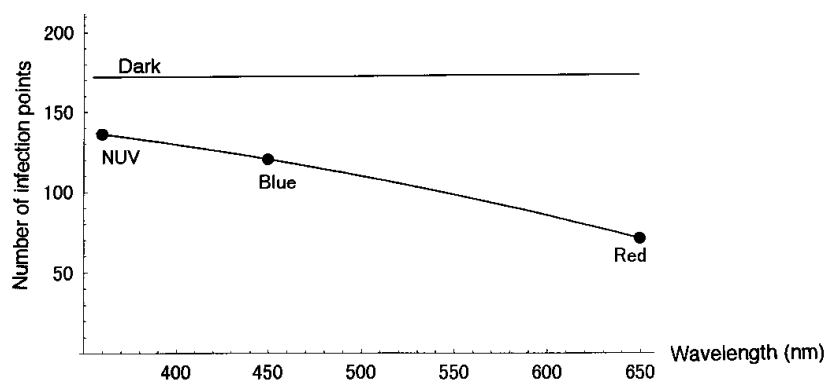

Fig. 10. Interpolating polynomial function analysis of infection points developed per drop of inoculated conidial suspension after $24 \mathrm{~h}$ of inoculation in light-pretreated broad bean leaflets by Botrytis cinerea.

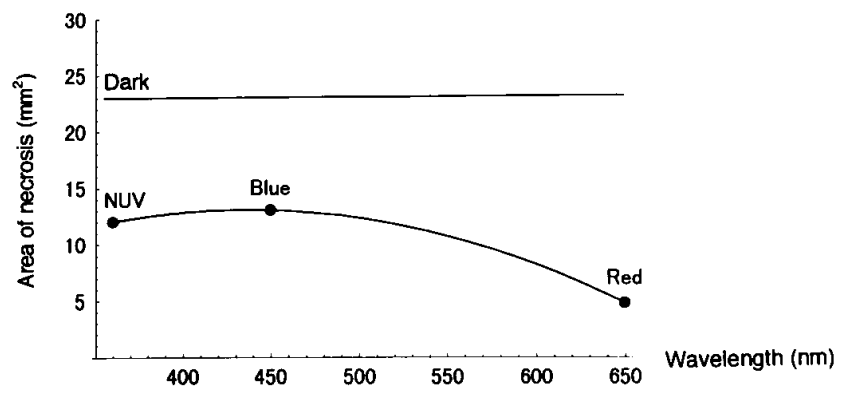

Fig. 11. Interpolating polynomial function analysis of necrosis development of Botrytis cinerea per drop of inoculated conidial suspension after $48 \mathrm{~h}$ of inoculation in light-pretreated broad bean leaflets.
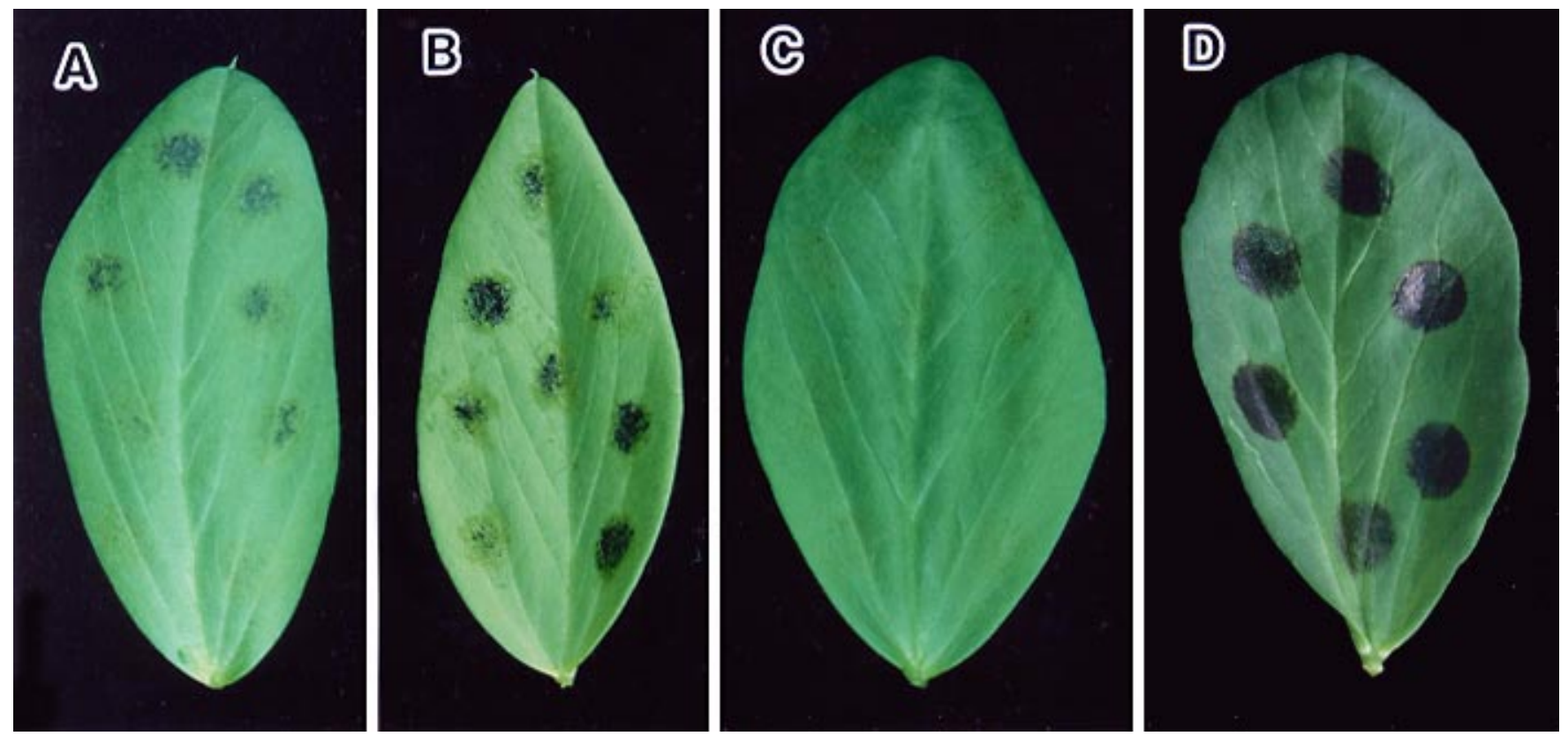

Fig. 12. Necrosis development of Botrytis cinerea in pretreated broad bean leaflets with the light of different wavelengths after $48 \mathrm{~h}$ of inoculation. (A) Pretreatment with NUV radiation, (B) pretreatment with blue light, (C) pretreatment with red light, and (D) pretreatment with dark. 


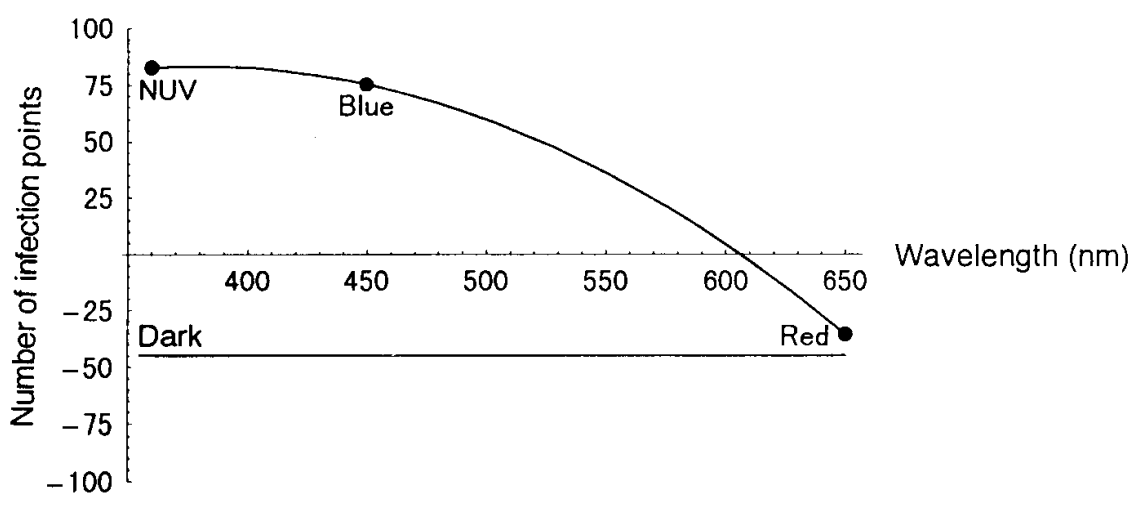

Fig. 13. Effect of germ tube-phototropism on infection points developed per drop of inoculated conidial suspension of Botrytis cinerea in broad bean leaf infection. Vertical axis shows the number of infection points obtained by deduction of data of pretreatment from post-treatment of leaflets.

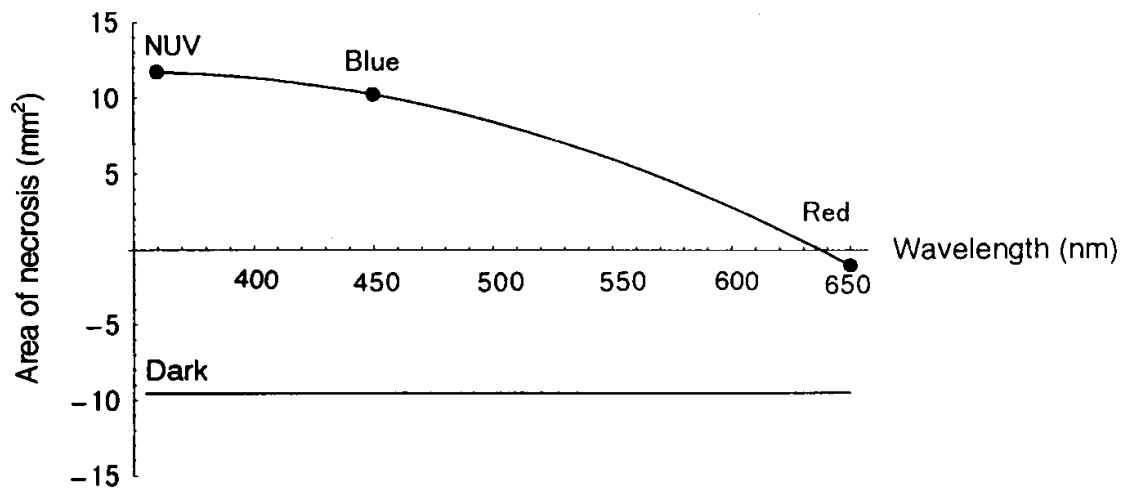

Fig. 14. Effect of germ tube-phototropism on necrosis development of Botrytis cinerea per drop of inoculated conidial suspension in broad bean leaf infection. Vertical axis shows the area of necrosis obtained by deduction of data of pretreatment from post-treatment of leaflets.

number of infection points was significantly higher than on the inoculated leaflets kept under red light without pretreatment. But the area of necrosis developed under red light did not differ significantly from that on leaflets pretreated with red light. In red light or red-light-pretreated leaflets, the area of necrosis was significantly smaller than that on leaflets kept under NUV radiation and blue light or in the dark. Suppression of lesion enlargement in leaflets under red light or in redlight-pretreated leaflets was not due to phototropic response of germ tubes, but there might be other effects of red light on the host leaf. In leaflets kept under red light and red-light-pretreated leaflets, we observed inhibition of infection-hypha formation from appressoria, and browning of cells in small areas around the appressoria was also observed. The inhibition of infection-hypha formation under red light suggests the possibility of red-light-induced resistance of broad bean against $B$. cinerea.

\section{ACKNOWLEDGMENTS}

We thank M. Yamasaki, Interdisciplinary Faculty of Science and Engineering, Department of Mathematics and Computer Science, Shimane University for suggestions and statistical analysis of data.

\section{LITERATURE CITED}

1. Blaauw, A. H. 1914. Licht und Wachstum Int. Z. Bot. 6:641-703.

2. Davis, P. J. 1975. Interpolation and Approximation. Dover Publications, New York.

3. Dey, P. K. 1933. Studies in the physiology of the appressorium of Colletotrichum gloeosporioides. Ann. Bot. (Lond.) 47:305-312.

4. Fromme, F. D. 1915. Negative heliotropism of the urediniospore germ tubes of Puccinia rhamni. Am. J. Bot. 2:82-85.

5. Honda, Y., Kashima, T., and Kumagai, T. 1992. Suppression of brown spot disease of cultivated chrysanthemum by manipulating phototropic response of conidium germ tubes of Septoria obesa. J. Phytopathol. 136:270-278.

6. Honda, Y., and Miyawaki, T. 1989. Phototropic response of conidium germ tubes in Septoria obesa. Trans. Mycol. Soc. Jpn. 30:411-414.

7. Honda, Y., and Yunoki, T. 1978. Action spectrum for photosporogenesis in Botrytis cinerea Pers. ex Fr. Plant Physiol. 61:711713.

8. Islam, S. Z., and Honda, Y. 1996. Influence of phototropic response of spore germ tubes on infection process in Colletotrichum lagenarium and Bipolaris oryzae. Mycoscience 37(3):331-337.

9. Jaffe, L., and Etzold, E. 1962. Orientation and locus of tropic photoreceptor molecules in spores of Botrytis and Osmunda. J. Cell Biol. 13:13-31.

10. Koch, E., and Hoppe, H. 1987. Effect of light on uredospore germination and germ tube growth of soybean rust (Phakopsora pachyrhizi Syd.). J. Phytopathol. 118:64-74.

11. Koga, K., Sato, T., and Ootaki, T. 1984 Negative phototropism in the piloboloid mutants of Phycomyces blakesleeanus Bgff. Planta 162:97-103.

12. Kumagai, T. 1988. Photocontrol of fungal development. Photochem. Photobiol. 47(6):889-896.

13. Last, F. T., and Hamley, R. E. 1956. A locallesion technique for measuring the infectivity of conidia of Botrytis fabae Sardina. Ann. Appl. Biol. 44(3):410-418.

14. Mercure, E. W., Kunoh, H., and Nicholson, R. L. 1994. Adhesion of Colletotrichum graminicola conidia to corn leaves: a requirement for disease development. Physiol. Mol. Plant Pathol. 45(6):407-420.

15. Nicholson, R. L. 1984. Adhesion of fungi to the plant cuticle. Pages 74-89 in: Infection Process of Fungi, D. W. Roberts and J. R. Aist, eds. Rockefeller Foundation, New York.

16. Nicholson, R. L., Kunoh, H., Shiraishi, T., and Yamada, T. 1993. Initiation of the infection process by Erysiphe graminis: conversion of the conidial surface from hydrophobicity to hydrophilicity and influence of the conidial exodate on the hydrophobicity of the barley leaf surface. Physiol. Mol. Plant Pathol. 43(4):307-318.

17. Staples, R. C., and Macko, V. 1980. Formation of infection structures as a recognition response in fungi. Exp. Mycol. 4:2-16.

18. Tan, K. K. 1978. Light induced fungal development. Pages 334-357 in: The Filamentous Fungi. J. E. Smith and D. R. Berry, eds. Edward Arnold Ltd., London.

19. Wilson, A. R. 1937. The chocolate spot disease of beans (Vicia faba L.) caused by $\mathrm{Bo}$ trytis cinerea Pers. Ann. Appl. Biol. 24:288

20. Xiao, J. Z., Watanabe, T., Kamakura, T., Ohshima, A., and Yamaguchi, I. 1994. Studies on cellular differentiation of Magnaporthe grisea. Physicochemical aspects of substratum surfaces in relation to appressorium formation. Physiol. Mol. Plant Pathol. 44:227-236.

21. Yarwood, C. E. 1932. Reversal phototropism of the germ tubes of clover powdery mildew. (Abstr.) Phytopathology 22:31 